http://dx.doi.org/10.1590/0102-4698122477

\title{
CARACTERÍSTICAS DO TRABALHO DE ESTUDANTES UNIVERSITÁRIOS ASSOCIADAS AO SEU DESEMPENHO ACADÊMICO
}

\author{
Roberta Pereira Niquini* \\ Liliane Reis Teixeira** \\ Clóvis Arlindo de Sousa*** \\ Roberta Nagai Manelli**** \\ Andréa Aparecida da Luz $* * * *$ \\ Samantha Lemos Turte-Cavadinha****** \\ Frida Marina Fischer $* * * * * *$
}

RESUMO: Embora se saiba da elevada prevalência de estudantes universitários trabalhadores, tem-se ainda evidências limitadas sobre as características do trabalho que influenciam o seu desempenho acadêmico. Assim, este estudo objetiva identificar as características do trabalho associadas ao desempenho acadêmico de universitários trabalhadores. Trata-se de um estudo transversal realizado em 2007/2008 com uma amostra de alunos de graduação de uma universidade pública $(n=211)$. Para estimar a associação entre as variáveis explicativas e o desempenho acadêmico, utilizou-se um modelo de regressão logística múltiplo. Apresentaram associação positiva com o baixo desempenho acadêmico: o sexo masculino; maiores jornada de trabalho e demanda; e baixos controle e apoio social no trabalho. Destacou-se a necessidade de atenção dos alunos e dos docentes na seleção de ambientes de trabalho/estágio potencialmente favoráveis ao melhor desempenho acadêmico.

Palavras-chave: Trabalhadores. Trabalho e estudo. Educação superior. Desempenho acadêmico. Autoavaliação.

\footnotetext{
* Especialista em Docência do Ensino Superior pela Universidade Gama Filho (UGF). Doutora em Ciências pelo Programa de Pós-graduação em Epidemiologia em Saúde Pública da Escola Nacional de Saúde Pública da Fundação Oswaldo Cruz (ENSP. FIOCRUZ). E-mail: roberta.niquini@icict.fiocruz.br

** Pós-doutora pela Faculdade de Saúde Pública da Universidade de São Paulo (FSP-USP). Pesquisadora do Centro de Estudos da Saúde do Trabalhador e Ecologia Humana da (ENSP- FIOCRUZ). E-mail: lilianeteixeira@ensp.fiocruz.br

* * Doutor em Ciências pelo Programa de Pós-Graduação em Saúde Pública da Faculdade de Saúde Pública da Universidade de São Paulo (USP). ProfessorTitular do Departamento de Educação Física e do Programa de Pós-Graduação em Saúde Coletiva da Universidade Regional de Blumenau (FURB). E-mail: clovissousa@usp.br

**** Doutora em Ciências pelo Programa de Pós-Graduação em Saúde Pública da FSP . USP. Pesquisadora do Departamento de Otorrinolaringologia, Instituto Karolinska, Estocolmo, Suécia. E-mail: roberta01@gmail.com

***** Doutoranda em Ciências pelo Programa de Pós Graduação em Saúde Pública da Faculdade de Saúde Pública da Universidade de São Paulo (USP). E-mail: andrealuz@usp.br

****** Doutoranda em Ciências pelo Programa de Pós-Graduação em Saúde Pública da Faculdade de Saúde Pública da Universidade de São Paulo (USP). E-mail: samturte@usp.br ******* Professora titular do Departamento de Saúde Ambiental da Faculdade Saúde Pública da Universidade de São Paulo (USP) e do Programa de Pós-Graduação em Saúde Pública da Faculdade de Saúde Pública (USP). E-mail: fischer.frida@gmail.com
} 


\section{WORK CHARACTERISTICS ASSOCIATED WITH COLLEGE STUDENTS' ACADEMIC PERFORMANCE}

ABSTRACT: Despite the high prevalence of working college students, there is limited evidence on the job characteristics that affect their academic performance. Thus, the aim of the present study is to identify job characteristics associated to the working college students' academic performance. A cross-sectional study was performed in 2007/2008, with a sample of undergraduate students from a public university ( $n=211)$. A multiple logistic regression model was used in order to estimate the association between the explanatory variables and academic performance. Results show that low academic performance was associated with: being male, longer working hours and higher demands, low control and low social support at work. This study has highlighted the need for drawing students and teachers' attention to the selection of jobs/internships that can positively influence their academic performance.

Keywords: Workers. Work and study. Higher education. Academic performance. Self-assessment.

\section{INTRODUÇÃO}

Estudos realizados em diversos países relataram prevalências entre 53\% e 89\% de estudantes universitários trabalhadores, com jornadas de trabalho médias de 15 horas semanais ou mais (BUTLER, 2007; COTTON et al., 2002; DEROUS; RYAN, 2008; FURR; ELLING, 2000; HAWKINS et al., 2005; MCKECHNIE et al., 2005; MILLER et al., 2008; NAGAI-MANELLI et al., 2012; ROCHFORD et al., 2009; SALAMONSON et al., 2012). No contexto brasileiro, pesquisas realizadas com estudantes do ensino médio de escolas públicas já têm evidenciado frequências entre $52 \%$ e $70 \%$ de discentes trabalhadores (FISCHER et al., 2003; OLIVEIRA et al., 2005), com pouco menos de um terço cumprindo jornadas diárias de trabalho acima de oito horas (FISCHER et al., 2003).

Embora se saiba dessa elevada prevalência de estudantes trabalhadores e de suas longas jornadas de trabalho, tem-se ainda evidências limitadas sobre as características do trabalho que prejudicam ou beneficiam o seu desempenho acadêmico (BUTLER, 2007; MCKECHNIE et al., 2005; ROCHFORD et al., 2009). Complementarmente, a literatura tem destacado uma tendência internacional de elevação da parcela de trabalhadores entre os estudantes do ensino superior, que parece vir acompanhada de maiores jornadas de trabalho, o que enfatiza, ainda mais, a 
necessidade de uma maior compreensão sobre como o trabalho afeta o desempenho desses estudantes (BUTLER, 2007; MCKECHNIE et al., 2005; SALAMONSON et al., 2012).

Os estudos nessa área são recentes, com concentração nos últimos 15 anos. O desfecho de interesse (desempenho acadêmico) tem sido obtido por meio de diversos métodos: registro do coeficiente de rendimento acumulado do aluno (COTTON et al., 2002; DEROUS; RYAN, 2008; SALAMONSON et al., 2012; TROCKEL et al., 2000); combinação desse coeficiente com outras medidas, como frequência às aulas (BUTLER, 2007); razão do número de disciplinas finalizadas pelo número de semestres cursados (CHAMBEL; CURRAL, 2005); nota média autorreferida (HAWKINS et al., 2005; MILLER et al., 2008); entre outras variáveis proxy (representantes) do desempenho acadêmico, como satisfação com a vida acadêmica (CHAMBEL; CURRAL, 2005), atitude de estudo (DEROUS; RYAN, 2008) e tempo gasto em sala de aula (NAGAI-MANELLI et al., 2012).

Associações negativas entre esses desfechos e as elevadas jornadas de trabalho dos estudantes universitários (DEROUS; RYAN, 2008; HAWKINS et al., 2005; MCKECHNIE et al., 2005; MILLER et al., 2008; NAGAI-MANELLI et al., 2012; ROCHFORD et al., 2009; SALAMONSON et al., 2012; TROCKEL et al., 2000) têm sido amplamente documentadas. Entretanto, há muitos outros fatores relacionados ao trabalho, menos explorados na literatura, que parecem afetar o desempenho acadêmico e suas variáveis proxy, como demanda, controle (BUTLER, 2007; CHAMBEL; CURRAL, 2005), apoio (CHAMBEL; CURRAL, 2005) e autonomia no trabalho (DEROUS; RYAN, 2008) e interações entre essas variáveis (CHAMBEL; CURRAL, 2005; DEROUS; RYAN, 2008). Além disso, variáveis demográficas têm sido apontadas como potenciais confundidoras dessa associação, como idade (COTTON et al., 2002; DEROUS; RYAN, 2008) e sexo do aluno (DEROUS; RYAN, 2008; HAWKINS et al., 2005; NAGAI-MANELLI et al., 2012).

Acredita-se que, a partir da identificação das características do trabalho relacionadas positivamente e negativamente ao desempenho acadêmico, em culturas e contextos de trabalho diferentes, os docentes do ensino superior possam modificar a política de escolha e aprovação dos estágios durante a graduação (MCKECHNIE et al., 2005; SALAMONSON et al., 2012); ampliar o acesso de seus alunos a essas evidências científicas e fomentar discussões acerca do tema para motivar a escolha consciente do estágio/trabalho pelos estudantes. E, ainda, desenvolver abordagens/estratégias em suas 
disciplinas para identificar os alunos em condições de trabalho/ estágio que os deixem mais vulneráveis a um pior aproveitamento nos estudos, de modo que os professores deem a eles maior apoio e estímulo, para maximizar seu desempenho acadêmico e reduzir o abandono do curso (TROCKEL et al., 2000).

Diante disso, este estudo tem o objetivo de identificar as características do trabalho associadas positivamente e negativamente ao desempenho acadêmico autorreferido de uma amostra de estudantes universitários trabalhadores, matriculados entre julho de 2007 e junho de 2008, nos cursos noturnos de Administração, Economia, Contabilidade ou Ciências Atuariais de uma universidade pública no Estado de São Paulo, Brasil.

\section{MÉTODO}

Trata-se de um subprojeto do estudo "Jovens universitários e as jornadas duplas de trabalho: repercussões na saúde e na qualidade de vida", desenvolvido pelo grupo de pesquisa Organização do Trabalho, Riscos e Prevenção à Saúde do Trabalhador/ Trabalho de jovens, representado por pesquisadores da Universidade de São Paulo (USP), com participação de pesquisadores da Escola Nacional de Saúde Pública Sérgio Arouca, da Fundação Oswaldo Cruz (Ensp/Fiocruz). Para a realização deste estudo, contou-se com o auxílio financeiro do Conselho Nacional de Desenvolvimento Científico e Tecnológico - CNPq (501766/2007-3; 500782/2008-3; 472153/2006-4; 307919/2006-4); da Fundação de Amparo à Pesquisa do Estado de São Paulo - Fapesp (07/04648-4; 06/59053-2); do Programa Institucional de Bolsas de Iniciação Científica - Pibic; e da Coordenação de Aperfeiçoamento de Pessoal de Nível Superior - Capes.

Foi realizado um estudo transversal entre 31 de julho de 2007 e 30 de junho de 2008 com uma amostra da população de estudantes universitários trabalhadores, com idades entre 18 e 26 anos, que cursavam qualquer período letivo, no turno da noite, de um dos quatro cursos oferecidos na Faculdade de Economia e Administração (FEA) (Administração, Economia, Contabilidade e Ciências Atuariais) de uma universidade pública no Estado de São Paulo, Brasil. Optou-se por não incluir menores de idade, devido às complexidades éticas. Dessa forma, a faixa etária de 18 a 26 anos foi escolhida por incluir os $80 \%$ mais jovens entre os universitários maiores de idade da FEA no período de estudo. 
O cálculo do tamanho amostral para esse subprojeto foi realizado assumindo-se nível de significância de $5 \%$, proporção de $50 \%$ e margem de erro de 0,065; o valor sofreu correção para a população finita ( $\mathrm{n}=1.165$ estudantes universitários pertencentes à populaçãoalvo), totalizando uma amostra de 191 estudantes trabalhadores. Dada a prevalência de estudantes universitários trabalhadores na FEA, estimada em no mínimo $85 \%$, segundo informação fornecida pelos alunos de todas as turmas no mês de junho de 2007, a amostra foi recalculada para 225 estudantes.

Os estudantes elegíveis da FEA foram convidados (pessoalmente, via internet e por veículos de comunicação escrita da FEA) a participar do estudo, que incluiu as respostas a "questionário de caracterização das condições de vida, saúde, sono e trabalho", de particular interesse neste subprojeto.

Transcorrido um ano de coleta de dados, obtiveram-se 227 participantes, os quais assinaram o Termo de Consentimento Livre e Esclarecido antes de iniciarem o preenchimento do questionário. Foram excluídos deste subprojeto todos os estudantes universitários que afirmaram não trabalhar $(\mathrm{n}=8 ; 3,5 \%)$, tendo sido considerado trabalho "toda atividade sistemática em que há uma obrigatoriedade de desenvolver tarefas, em horários e períodos pré-determinados, seja no ambiente doméstico, seja para terceiros, com ou sem remuneração, tendo ou não vínculo empregatício formalizado", conforme descrito por Fischer et al. (2003, p. 975). Um aluno foi excluído por não informar seu desempenho acadêmico, e outros sete, por não fornecerem informações sobre seu trabalho (jornada, demanda, controle e apoio social), totalizando-se uma amostra final de 211 estudantes trabalhadores.

As questões presentes no "questionário de caracterização das condições de vida, saúde, sono e trabalho" sobre dados demográficos (idade atual e sexo) e de trabalho (idade em que entrou no mercado de trabalho, registro formal de trabalho, ramo de atividade, jornada de trabalho e renda habitual) foram extraídas de protocolos utilizados em estudo anterior (FISCHER et al., 2003).

A escala utilizada para verificar demanda, controle e apoio social no trabalho foi uma versão reduzida da "job stress scale", adaptada para o português por Alves et al. (2004). Nesse modelo proposto, entendem-se por demanda as pressões de natureza psicológica, como tempo e ritmo na realização do trabalho e conflito entre as demandas. Já o controle relaciona-se ao uso de habilidades 
intelectuais no trabalho e à autoridade para tomar decisões sobre como realizar as atividades necessárias. Por fim, o apoio social é compreendido como o grau de interação social com colegas e superiores (ALVES et al., 2004).

Seguindo a lógica desse modelo teórico, são nocivos ao trabalhador: as altas demandas combinadas com o baixo controle no processo de trabalho (porque podem gerar desgaste) e as baixas demandas combinadas com o baixo controle (porque podem gerar desinteresse). A situação "ideal" seria a combinação de baixas demandas com alto controle do processo de trabalho. Por sua vez, o baixo apoio social no ambiente de trabalho é visto como nocivo, independentemente das outras duas dimensões (ALVES et al., 2004).

A escala utilizada é composta de 17 questões, sendo cinco para avaliar demanda - Com que frequência você tem que fazer suas tarefas de trabalho com muita rapidez? Com que frequência você tem que trabalhar intensamente (isto é, produzir muito em pouco tempo)? Seu trabalho exige demais de você? Você tem tempo suficiente para cumprir todas as tarefas de seu trabalho? O seu trabalho costuma apresentar exigências contraditórias ou discordantes? -, seis sobre controle - Você tem possibilidade de aprender coisas novas em seu trabalho? Seu trabalho exige muita habilidade ou conhecimentos especializados? Seu trabalho exige que você tome iniciativas? No seu trabalho, você tem que repetir muitas vezes as mesmas tarefas? Você pode escolher como fazer o seu trabalho? Você pode escolher o que fazer no seu trabalho? - e seis sobre apoio social - Existe um ambiente calmo e agradável onde trabalho. No trabalho, nos relacionamos bem uns com os outros. Eu posso contar com o apoio dos meus colegas de trabalho. Se eu não estiver num bom dia, meus colegas compreendem. No trabalho, eu me relaciono bem com meus chefes. Eu gosto de trabalhar com meus colegas. As opções de respostas nas duas primeiras dimensões variavam de "frequentemente" a "nunca" e, na terceira, de "concordo totalmente" a "discordo totalmente", tendo sido atribuídos de 1 a 4 pontos para cada resposta, sendo a maior pontuação associada à maior demanda ou ao maior controle ou ao maior apoio social no trabalho (ALVES et al., 2004).

A variável desempenho acadêmico foi obtida por meio da seguinte questão: "Marque, em uma escala de 0 a 10, que nota você daria a seu próprio desempenho acadêmico". A autoavaliação do desempenho acadêmico tem revelado correlação positiva significativa com a avaliação dos professores (LÓPEZ-PASTOR et 
al., 2012; KARNILOWICZ, 2012). Permite o reconhecimento dos estudantes como pessoas ativas, responsáveis e reflexivas, envolvidas no julgamento de seu próprio aprendizado e dos seus resultados de aprendizagem (DOCHY et al., 1999), além de incorporar, em parte, as opiniões e os julgamentos dos pares, já que vivemos em comunidade, compartilhando valores (NULTY, 2011).

O pré-teste desse questionário foi realizado durante o mês de junho de 2007, com alunos que estudavam no período noturno no curso de Nutrição da Faculdade de Saúde Pública da mesma universidade, permitindo estimar seu tempo de preenchimento (aproximadamente 30 minutos) e verificar as dúvidas, as dificuldades e os erros, que sofreram correção antes do início da coleta de dados. O questionário foi autopreenchido online ou na forma impressa (de acordo com a disponibilidade/opção do aluno).

$\mathrm{Na}$ análise de dados deste subprojeto, inicialmente, foram apresentadas as frequências absolutas e relativas das variáveis categóricas e as medidas de tendência central (média e mediana) e de dispersão (desvio padrão) das variáveis contínuas. Antes de proceder às análises de regressão logística simples, foi testada a normalidade da distribuição das variáveis contínuas por meio do teste KolmogorovSmirnov, adotando-se um nível de significância de 5\%. Para analisar o relacionamento linear entre as variáveis contínuas, foi utilizado o coeficiente de correlação de Pearson (r), no caso de as variáveis terem distribuição normal, e o coeficiente de correlação de Spearman (rho), caso contrário. As variáveis contínuas que não se associaram linearmente ao desempenho acadêmico, com significância de 5\%, foram categorizadas para proceder à regressão logística simples.

Foram realizadas regressões logísticas simples entre a variável dependente desempenho acadêmico $(0$ - nota $\geq 7$ pontos e 1 - nota $<7$ pontos, sendo 7,0 a média adotada como ponto de corte na universidade para a aprovação direta nas disciplinas, sem necessidade de prova final) e cada uma das variáveis independentes contínuas (idade atual, idade em que entrou no mercado de trabalho, jornada diária de trabalho, remuneração habitual) e categóricas sexo (0- feminino / 1 - masculino), ramo de atividade (0- instituição de pesquisa ou ensino / 1- outras (escritório, indústria, loja, buffet, hotel, academia, artes, própria casa e casa de terceiros)) e registro formal de trabalho (0- não/ 1 - sim). As variáveis independentes demanda, controle e apoio social no trabalho foram analisadas tanto na forma contínua como na dicotomizada, utilizando-se a mediana 
como ponto de corte (nível baixo/alto). Foram calculados as estimativas de odds ratio (OR) brutas de associação entre cada uma das variáveis independentes e a variável desempenho acadêmico e seus respectivos intervalos de confiança (com 95\% de confiança). Posteriormente, foram testadas seis interações, de acordo com a literatura (CHAMBEL; CURRAL, 2005; DEROUS; RYAN, 2008): demanda com controle/apoio/jornada de trabalho, controle com apoio/jornada de trabalho e apoio com jornada de trabalho.

As variáveis e as interações com significância menor que 20\% na análise de regressão logística simples tiveram a sua inclusão testada no modelo múltiplo. Foi realizada, então, a seleção de variáveis independentes por meio do procedimento de stepwise manual, tomando-se o cuidado para não manter, no modelo, as variáveis categóricas e contínuas que representassem o efeito da mesma variável original. Foram mantidas no modelo múltiplo de regressão logística final as variáveis independentes e as interações significativas para explicar o desempenho acadêmico no nível de 5\%; os efeitos principais das variáveis que apresentaram interação; e as variáveis independentes com significância limítrofe (entre 5\% e 10\%), mas com relevância já bem-descrita na literatura.

Para avaliar a adequação do modelo, foi realizado o teste Hosmer e Lemeshow, bem como a análise de resíduos, por meio do gráfico "normal Q-Q plot com envelope", para a verificação da normalidade dos resíduos, e do gráfico de resíduo deviance padronizado, para verificar a presença de tendência na distribuição dos resíduos e de pontos influentes. Após a definição do melhor modelo, foram calculadas as estimativas de odds ratio (OR) ajustadas e os respectivos Intervalos de Confiança - ICs (com 95\% de confiança).

Para as interações (entre duas variáveis independentes) significativas para explicar o desempenho acadêmico, a associação de uma dessas variáveis independentes com o desfecho de interesse foi estimada por meio do cálculo do seu OR e do IC 95\%, segundo estratos da outra variável independente. Foram, ainda, elaborados gráficos com as probabilidades ajustadas de baixo desempenho acadêmico, segundo as duas variáveis independentes que apresentaram interação. As análises foram realizadas no software $R$ versão 2.14.0.

A pesquisa foi conduzida dentro dos padrões exigidos pela Declaração de Helsinque e aprovada pelo Comitê de Ética em Pesquisa da Faculdade de Saúde Pública da USP (Of. COEP/139/07). Não houve conflito de interesse em relação aos métodos utilizados como parte da investigação ou interesse financeiro dos pesquisadores. 


\section{RESULTADOS}

Entre os 219 estudantes universitários trabalhadores que responderam o questionário, $211(96,3 \%)$ forneceram as informações sobre o trabalho e o desempenho acadêmico, de interesse neste estudo. Nesta amostra de alunos, 55,5\% $(\mathrm{n}=116)$ eram do sexo masculino, com média e mediana de idade de 22 anos e desvio padrão (DP) de 2,0 anos.

Quanto às características de trabalho, 55,5\% $(\mathrm{n}=116)$ trabalhavam com registro formal (carteira assinada), e, quanto ao ramo de atividade, $80,6 \%(\mathrm{n}=170)$ trabalhavam em escritório; $12,3 \%$ $(n=26)$, em instituições de pesquisa ou ensino; $3,3 \%(n=7)$, na indústria, 2,4\% ( $\mathrm{n}=5)$, em loja, buffet, hotel, academia ou artes; e $1,4 \%(n=3)$ na própria casa/casa de terceiros.

Tanto a média quanto a mediana da idade de entrada no mercado de trabalho foram de 19 anos $(\mathrm{DP}=2,3)$, e as da jornada diária de trabalho foram de 8 horas $(\mathrm{DP}=2,1)$. A mediana da remuneração habitual foi de $R \$ 1.200,00$, enquanto a média foi de $\mathrm{R} \$ 1.538,79(\mathrm{DP}=1.407,60)$, revelando a assimetria à direita da distribuição. A média e a mediana foram iguais para a demanda (13 pontos; $\mathrm{DP}=2,9)$ e o apoio social no trabalho (20 pontos; $\mathrm{DP}=3,0)$ e próximas para o controle no trabalho (mediana $=18$; média $=17$; $\mathrm{DP}=2,9)$. Para o desempenho acadêmico, a média e a mediana foram de sete pontos $(\mathrm{DP}=1,4)$.

Todas as variáveis contínuas analisadas (desempenho acadêmico, idade atual, idade de entrada no mercado de trabalho, remuneração habitual, jornada diária de trabalho, demanda, controle e apoio social no trabalho) apresentaram distribuição significativamente (no nível de 5\%) diferente da normal, segundo o teste de Kolmogorov-Smirnov.

O desempenho acadêmico apresentou correlação linear significativa no nível de 5\% com todas as variáveis contínuas analisadas, exceto com a idade de entrada no mercado de trabalho, que, para a análise de regressão logística simples, foi dicotomizada em menor de 19 anos e com 19 anos ou mais, utilizando-se a mediana como ponto de corte. $\mathrm{O}$ desempenho acadêmico correlacionouse negativamente com idade atual, remuneração habitual, jornada diária e demanda no trabalho; e positivamente com o controle e o apoio social no trabalho (Tabela 1). 
TABELA 1 - Coeficiente de Correlação de Spearman (rho) entre as variáveis contínuas demográficas e do trabalho de estudantes universitários. São Paulo, SP, 2007/2008.

\begin{tabular}{|c|c|c|c|c|c|c|c|c|}
\hline Variáveis & Idade atual & $\begin{array}{c}\text { Idade de } \\
\text { entrada no } \\
\text { mercado }\end{array}$ & $\begin{array}{c}\text { Remuneração } \\
\text { habitual }\end{array}$ & $\begin{array}{c}\text { Jornada } \\
\text { diária }\end{array}$ & Demanda & Controle & $\begin{array}{l}\text { Apoio } \\
\text { social }\end{array}$ & $\begin{array}{c}\text { Desempenho } \\
\text { acadêmico }\end{array}$ \\
\hline Idade atual & 1,000 &, $233^{* *}$ &, $435^{* *}$ &, $287^{* *}$ & ,219** & 117 & , $160^{*}$ & $-189^{* *}$ \\
\hline $\begin{array}{l}\text { Idade de } \\
\text { entrada no } \\
\text { mercado }\end{array}$ &, $233 * *$ & 1,000 & -078 & , 013 & -009 & ,088 & ;,060 & ,036 \\
\hline $\begin{array}{l}\text { Remuneração } \\
\text { habitual }\end{array}$ &, $435^{* *}$ & -078 & 1,000 &, $574^{* *}$ &, $276^{* *}$ & , 122 & $-186^{* *}$ & $-237^{* *}$ \\
\hline $\begin{array}{l}\text { Jornada } \\
\text { diária }\end{array}$ &, $287^{* *}$ & -013 &, $574 * *$ & 1,000 &, $223^{* *}$ &, $164^{*}$ & -114 & $-152^{*}$ \\
\hline Demanda & ,219** & ,009 &, $276^{* *}$ &, $223^{* *}$ & 1,000 & ,066 & $-386^{* *}$ & $-198^{* *}$ \\
\hline Controle & 117 & -088 & , 122 &, $164^{*}$ & ,066 & 1,000 &, $246 * *$ &, $137^{*}$ \\
\hline Apoio social & $-160^{*}$ & -060 & -186 ** & -,114 &,$- 386^{* *}$ &, $246^{* *}$ & 1,000 &, $262^{* *}$ \\
\hline $\begin{array}{l}\text { Desempenho } \\
\text { acadêmico }\end{array}$ & -189** & ,036 & $-237^{* *}$ & $-152^{*}$ & $-198^{* *}$ &, $137^{*}$ &, $262^{* *}$ & 1,000 \\
\hline
\end{tabular}

$\mathrm{Na}$ Tabela 2, observa-se que o desempenho acadêmico mostrou associação significativa, no nível de $20 \%$, com todas as características demográficas e do trabalho analisadas, exceto com a idade de entrada no mercado de trabalho. Apresentaram associação positiva com o baixo desempenho acadêmico: a maior idade atual, o sexo masculino, ter registro formal de trabalho, trabalhar em ramo de atividade diverso de instituições de ensino e pesquisa, maiores jornada de trabalho, remuneração habitual e demanda no trabalho e baixos controle e apoio social no trabalho.

A única interação significativa, no nível de $20 \%$, para explicar o desempenho acadêmico foi a apresentada entre controle no trabalho e jornada diária de trabalho. 
TABELA 2 - Análise não ajustada das características demográficas e do trabalho associadas ao baixo desempenho acadêmico entre estudantes universitários. São Paulo, SP, 2007/2008.

\begin{tabular}{|c|c|c|}
\hline Variáveis & $\mathbf{O R}$ & IC 95\% \\
\hline Idade atual $\dagger$ & $1,15^{*}$ & $0,99-1,33$ \\
\hline \multicolumn{3}{|l|}{ Sexo } \\
\hline Feminino & 1 & \\
\hline Masculino & $1,72^{*}$ & $0,96-3,06$ \\
\hline \multicolumn{3}{|c|}{ Idade de entrada no mercado de trabalho } \\
\hline$\geq 19$ anos & 1 & \\
\hline$<19$ anos & 1,08 & $0,60-1,91$ \\
\hline \multicolumn{3}{|l|}{ Registro formal de trabalho } \\
\hline Não & 1 & \\
\hline Sim & $1,53^{*}$ & $0,86-2,72$ \\
\hline \multicolumn{3}{|l|}{ Ramo de atividade } \\
\hline Instituição de pesquisa ou ensino & 1 & \\
\hline Outro & $4,78^{* *}$ & $1,38-16,49$ \\
\hline Jornada diária de trabalho $†$ & $1,19^{* *}$ & $1,03-1,38$ \\
\hline Remuneração habitual † & $1,00^{*}$ & $1,00 \cdot 1,00$ \\
\hline Demanda no trabalho $\dagger$ & $1,21 * * *$ & $1,09-1,35$ \\
\hline \multicolumn{3}{|l|}{ Demanda no trabalho } \\
\hline Baixa & 1 & \\
\hline Alta & $2,01 * *$ & $1,10-3,67$ \\
\hline Controle no trabalho $\dagger$ & $0,90^{*}$ & $0,82-1,00$ \\
\hline \multicolumn{3}{|l|}{ Controle no trabalho } \\
\hline Alto & 1 & \\
\hline Baixo & $1,64^{*}$ & $0,92 \cdot 2,89$ \\
\hline Apoio no trabalho $\dagger$ & $0,82^{* * *}$ & $0,74-0,91$ \\
\hline \multicolumn{3}{|l|}{ Apoio no trabalho } \\
\hline Alto & 1 & \\
\hline Baixo & $3,11 * * *$ & $1,73-5,61$ \\
\hline
\end{tabular}

O melhor modelo testado na análise de regressão logística múltipla para explicar o baixo desempenho acadêmico (nota $<7$ pontos) foi o que incluía: as variáveis jornada diária de trabalho (contínua), demanda no trabalho (contínua), apoio social e controle no trabalho (categóricas); a interação entre as categorias de controle no trabalho e jornada diária de trabalho (contínua), significativas no nível de 5\%; 
e a variável sexo, com significância limítrofe, mas já reconhecida na literatura (DEROUS; RYAN, 2008; HAWKINS et al., 2005; NAGAIMANELLI et al., 2012) como associada ao desempenho acadêmico.

Na análise ajustada dos fatores associados ao baixo desempenho acadêmico (Tabela 3), observou-se que os alunos do sexo masculino tiveram uma chance $77,9 \%$ maior de baixo desempenho acadêmico do que as do sexo feminino (OR = 1,78; IC 95\%: 0,94 - 3,38), ou seja, os homens tiveram maior chance de referir notas abaixo de sete.

TABELA 3 - Análise ajustada das características demográficas e do trabalho associadas ao baixo desempenho acadêmico. São Paulo, SP, 2007/2008.

\begin{tabular}{|c|c|c|}
\hline Variáveis & OR & IC 95\% \\
\hline \multicolumn{3}{|l|}{ Sexo } \\
\hline Feminino & 1 & \\
\hline Masculino & $1,78^{*}$ & $0,94-3,38$ \\
\hline Demanda no trabalho $\dagger$ & $1,14^{* *}$ & $1,01 \cdot 1,29$ \\
\hline \multicolumn{3}{|l|}{ Apoio no trabalho } \\
\hline Alto & 1 & \\
\hline Baixo & $2,62 * * *$ & $1,33-5,15$ \\
\hline \multicolumn{3}{|c|}{ Para alunos com jornada diária de 4 horas } \\
\hline Alto controle no trabalho & 1 & \\
\hline Baixo controle no trabalho & $8,72^{* * *}$ & $1,73-43,98$ \\
\hline \multicolumn{3}{|c|}{ Para alunos com jornada diária de 6 horas } \\
\hline Alto controle no trabalho & 1 & \\
\hline Baixo controle no trabalho & $3,80^{* * *}$ & $1,37-10,53$ \\
\hline \multicolumn{3}{|c|}{ Para alunos com jornada diária de 8 horas } \\
\hline Alto controle no trabalho & 1 & \\
\hline Baixo controle no trabalho & 1,66 & $0,85-3,23$ \\
\hline \multicolumn{3}{|c|}{ Para alunos com jornada diária de 10 horas } \\
\hline Alto controle no trabalho & 1 & \\
\hline Baixo controle no trabalho & 0,72 & $0,29 \cdot 1,81$ \\
\hline
\end{tabular}

Legenda: *P-valor =0,08; * P-valor $<0,05 ;{ }^{* * *}$-valor $<0,01$; † Variável contínua.

Fonte: Elaboração pelos autores. 
Verificou-se que, para cada aumento de uma unidade na escala de demanda, os alunos apresentaram chance de baixo desempenho acadêmico $14,1 \%$ maior do que os alunos com a pontuação de demanda imediatamente inferior (OR=1,14; IC 95\%: 1,01 - 1,29), ou seja, a maior demanda no trabalho se revelou como um fator prejudicial ao desempenho acadêmico. Os estudantes com baixo apoio social no trabalho tiveram chance 2,62 vezes maior para o baixo desempenho acadêmico quando comparados aos estudantes com alto apoio social no trabalho (IC 95\%: 1,33-5,15). Observa-se, desse modo, que o alto apoio social no trabalho apresentou-se como fator que pode contribuir de forma positiva para o desempenho acadêmico dos jovens.

Para interpretar a interação entre as variáveis jornada de trabalho e controle no trabalho, as medidas de associação entre controle no trabalho e desempenho acadêmico foram apresentadas segundo estratos da variável jornada de trabalho. Dessa forma, para alunos com jornada diária de trabalho de quatro horas, os com baixo controle no trabalho apresentaram uma chance 8,72 vezes maior de baixo desempenho acadêmico do que aqueles com alto controle no trabalho (IC 95\%: 1,73-43,98); enquanto, entre os estudantes com jornada diária de trabalho de seis horas, os com baixo controle no trabalho apresentaram chance 3,80 vezes maior de baixo desempenho acadêmico do que os com alto controle no trabalho (IC 95\%: 1,3710,53). Essa associação positiva significativa entre o baixo controle no trabalho e o baixo desempenho acadêmico não foi observada para os estudantes com jornadas diárias de trabalho de oito e dez horas.

A presença de interação entre controle no trabalho e jornada diária de trabalho para explicar o baixo desempenho acadêmico dos alunos também pode ser verificada na Figura 1. Observou-se claramente que, entre os alunos com menos de oito horas diárias de trabalho, aqueles com baixo controle no trabalho têm maior probabilidade de baixo desempenho acadêmico. Também se verificou que, entre os estudantes com oito ou mais horas de trabalho, o desempenho acadêmico pareceu ser independente do controle no trabalho. Além disso, observou-se a associação positiva de maior jornada de trabalho com o baixo desempenho acadêmico entre os alunos com alto controle no trabalho, enquanto para os estudantes com baixo controle esse efeito não é percebido.

O modelo múltiplo de regressão logística final mostrou-se adequado segundo o teste de Hosmer e Lemeshow ( $\mathrm{p}$-valor=0,11). Quanto à análise de resíduos, como pode ser observado na Figura 2, os resíduos apresentaram distribuição aproximadamente normal, segundo o 
normal Q-Q plot com envelope, e ausência de tendência na distribuição e de pontos discordantes, segundo o gráfico de resíduo deviance padronizado, de forma que o modelo foi considerado adequado.

FIGURA 1 - Probabilidades de baixo desempenho acadêmico segundo jornada diária e controle no trabalho ajustados. São Paulo, SP, 2007/2008.

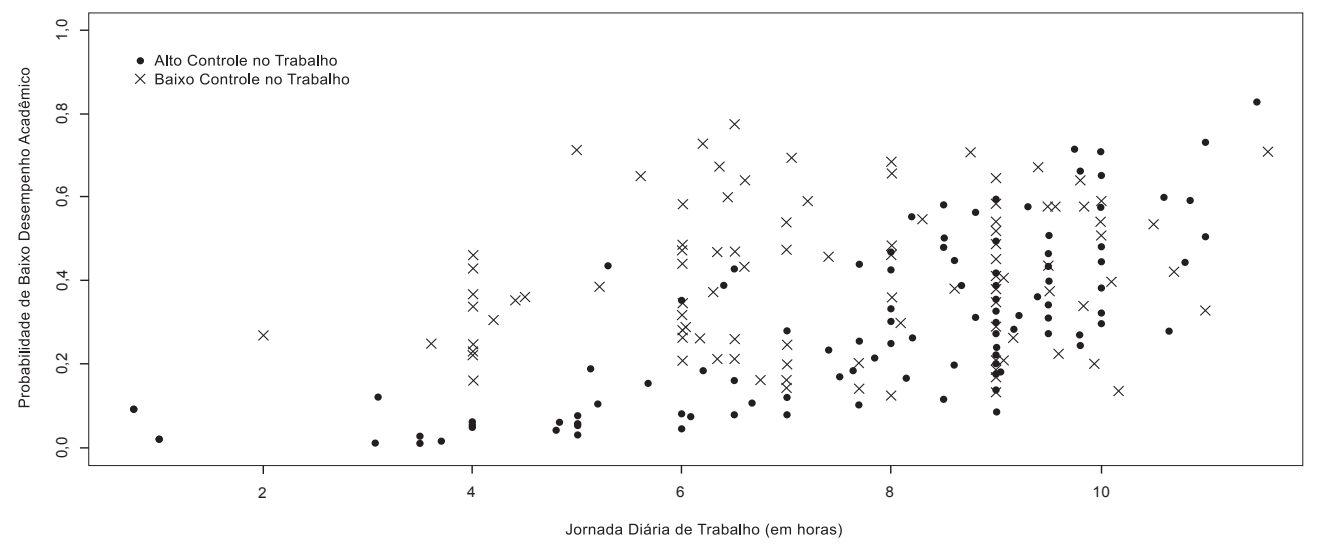

Fonte: Gráfico elaborado com o software $R$ versão 2.14 .0 com dados da pesquisa.

FIGURA 2 - Análise de Resíduos para o Modelo Ajustado. São Paulo, SP, 2007/2008.
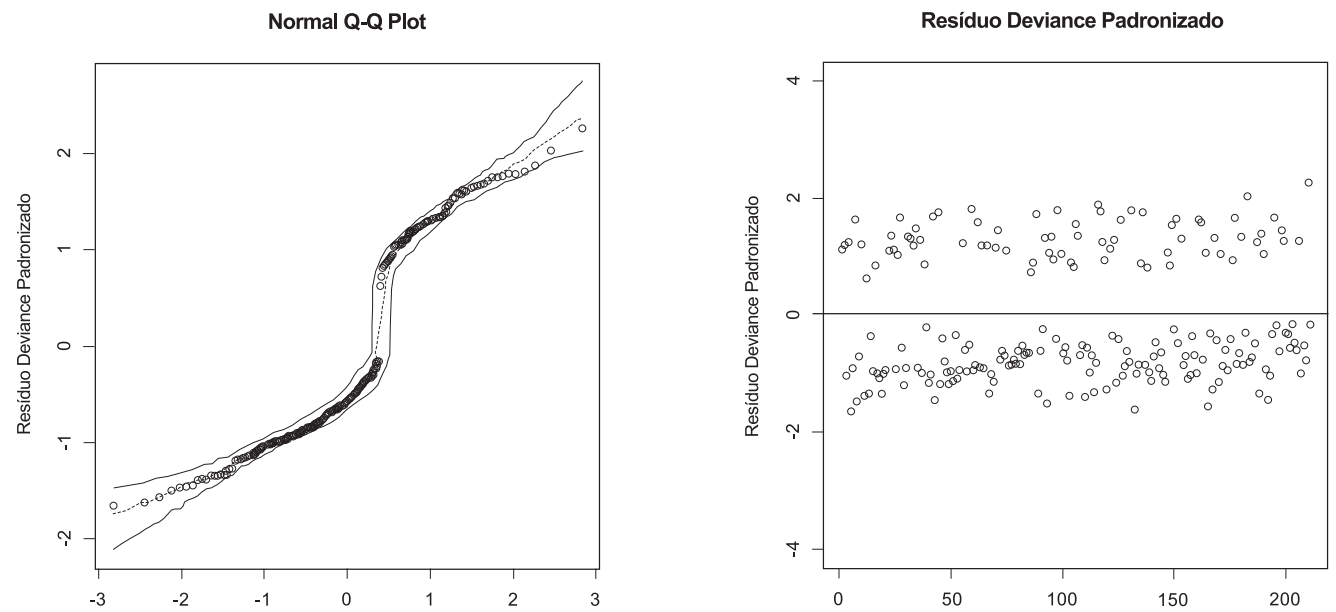

Fonte: Gráfico elaborado com o software R versão 2.14 .0 com dados da pesquisa. 


\section{DISCUSSÃO}

Apesar de outros autores já terem utilizado notas autorreferidas para medir o desempenho acadêmico de estudantes universitários (HAWKINS et al., 2005; MILLER et al., 2008), este estudo difere dos demais por ter obtido a nota que o aluno atribui ao seu desempenho acadêmico nas condições atuais de sua vida e trabalho, não tendo sido solicitado que o estudante reproduzisse a nota média acumulada obtida na universidade.

Ao se adotar a autoavaliação do desempenho como medida de desfecho, neste estudo, enfatizou-se o reconhecimento dos alunos como sujeitos reflexivos, responsáveis e ativos no julgamento de seu próprio aprendizado (DOCHY et al., 1999). Ressalta-se ainda que a autoavaliação tem sido vista como positiva na prática educacional (DOCHY et al., 1999). Assim, entende-se que o Ensino Superior é um palco oportuno para a busca da reflexão pelos alunos sobre seu aprendizado e para o desenvolvimento das habilidades de identificar critérios e de aplicá-los em julgamentos próprios, visando ao reconhecimento, ao controle e à responsabilidade sobre o próprio aprendizado e o desenvolvimento pessoal e profissional (DOCHY et al., 1999; NULTY, 2011).

Apesar de a literatura destacar que a autoavaliação do desempenho acadêmico correlaciona-se positivamente com a avaliação dos professores (KARNILOWICZ, 2012; LÓPEZPASTOR et al., 2012; NULTY, 2011), ao se utilizar a avaliação do professor como padrão-ouro, têm sido observados os seguintes vieses: maior correlação no grupo de alunos com melhor desempenho acadêmico; tendência de subestimação da nota pelos alunos de melhor desempenho; e superestimação pelos alunos de pior desempenho (KARNILOWICZ, 2012). Esse fato não foi considerado aqui como limitação da medida, tendo-se em vista que não se buscou a nota atribuída pelo professor, e, sim, a nota atribuída pelo aluno ao seu próprio desempenho acadêmico. Tal medida, entretanto, não é estritamente comparável com a obtida por outros autores.

Além disso, têm sido encontradas mais características do trabalho associadas significativamente com variáveis como atitude de estudo e satisfação com a vida acadêmica do que com o coeficiente de rendimento acumulado do aluno na universidade ou com a razão do número de disciplinas finalizadas pelo número de semestres cursados (CHAMBEL; CURRAL, 2005; DEROUS; RYAN, 2008). Isso tem sido explicado pelo fato de a capacidade 
cognitiva (não mensurada) ser um forte preditor do coeficiente de rendimento acumulado e das aprovações do aluno na universidade (DEROUS; RYAN, 2008), mesmo quando o aluno não julga que seu desempenho, seu aprendizado e seu aproveitamento no curso universitário estejam satisfatórios.

Alguns críticos podem argumentar que um pior desempenho acadêmico não é de grande importância para os estudantes concluintes do curso e até que os empregadores podem valorizar mais a experiência de trabalho do que o desempenho acadêmico, conforme ressaltado por Salamonson et al. (2012). Entretanto, tem sido ressaltada a menor frequência, entre estudantes trabalhadores, de relatos de que os professores os encorajam a interagir em atividades de classe ou que estão disponíveis para responder questões extraclasse e de que as relações estabelecidas com a universidade os ajudam a permanecer no curso (FURR; ELLING, 2000). Esse contexto pode resultar em, entre os estudantes trabalhadores, sobretudo entre aqueles em condições menos favoráveis, menor motivação com os estudos, formação mais limitada, prejuízo no aprendizado e na progressão normal do curso e até no abandono da graduação. Vale ressaltar que se estima que um terço dos alunos ingressantes em cursos noturnos ofertados em Instituições Federais de Educação Superior no Brasil não consiga alcançar a conclusão do curso, o que é uma fração bastante elevada, dada a gratuidade do ensino. Esse abandono tem origem multicausal, entretanto, a hipótese da situação de trabalho como obstáculo à educação superior deve ser considerada com atenção (VARGAS; DE PAULA, 2013).

Como limitação deste estudo, ressalta-se o fato de ter sido utilizado um desenho transversal capturando todas as informações no mesmo período do tempo, de maneira que não se pode assumir aqui a indicação da causalidade. No entanto, apesar de suas limitações, este desenho tem sido o mais utilizado em pesquisas com objetivos similares ao deste estudo (BUTLER, 2007; CHAMBEL; CURRAL, 2005; COTTON et al., 2002; DEROUS; RYAN, 2008; FURR; ELLING, 2000; HAWKINS et al., 2005; MCKECHNIE et al., 2005; MILLER et al., 2008; NAGAI-MANELLI et al., 2012; ROCHFORD et al., 2009; TROCKEL et al., 2000), não sendo limitada, portanto, a comparabilidade de seus resultados.

Neste estudo, o sexo masculino mostrou-se positivamente associado a um pior desempenho acadêmico. Tal resultado também foi observado em universidades públicas nos Estados Unidos por Hawkins et al. (2005), que também avaliaram o desempenho 
acadêmico autorreferido, em uma pesquisa com estudantes do curso de Serviço Social, e por Derous e Ryan (2008), que avaliaram a atitude de estudo entre alunos de Psicologia.

Ressalta-se que a população avaliada no presente estudo foi predominantemente do sexo masculino, diferentemente do que tem sido observado na maior parte das pesquisas que abordam esse tema, que apresentam percentuais entre $61 \%$ e $92 \%$ de alunos do sexo feminino (BUTLER, 2007; CHAMBEL; CURRAL, 2005; COTTON et al., 2002; DEROUS; RYAN, 2008; HAWKINS et al., 2005; MILLER et al., 2008; ROCHFORD et al., 2009; SALAMONSON et al., 2012). Essa diferença pode ter ocorrido devido aos cursos de graduação avaliados neste estudo (Administração, Economia, Contabilidade ou Ciências Atuariais) e os avaliados em muitas das outras pesquisas, tais como Enfermagem (ROCHFORD et al., 2009; SALAMONSON et al., 2012), Psicologia (CHAMBEL; CURRAL, 2005; DEROUS; RYAN, 2008), Serviço Social (HAWKINS et al., 2005) e Biologia (CHAMBEL; CURRAL, 2005), já que é reconhecida a predominância de estudantes/profissionais do sexo feminino na área de saúde.

Diferentemente do observado por Derous e Ryan (2008), neste estudo a idade não se mostrou significativamente e positivamente associada ao desempenho acadêmico. Pelo contrário, observou-se uma correlação negativa. Da mesma forma, Cotton et al. (2002) encontraram correlação negativa entre idade e carga de estudo, em uma amostra de estudantes universitários australianos. Por sua vez, no presente estudo, a idade apresentou correlação significativa positiva com jornada e demanda no trabalho (variáveis que se mostraram negativamente associadas ao desempenho acadêmico na análise múltipla), ou seja, alunos mais velhos tenderam a ter maiores jornada e demanda no trabalho. A idade apresentou, ainda, correlação significativa negativa com apoio social no trabalho (que foi positivamente associado ao desempenho acadêmico na análise múltipla). Essas relações observadas podem ter acarretado a correlação significativa negativa observada entre idade e desempenho acadêmico. Similarmente, Cotton et al. (2002) observaram uma correlação positiva entre idade e demanda no trabalho, e Chambel e Curral (2005), em um estudo realizado com estudantes universitários portugueses dos cursos de Biologia, Matemática e Psicologia, verificaram a correlação significativa negativa da idade com o apoio no trabalho.

Quanto à comparabilidade da idade da população analisada neste estudo com a observada em pesquisas que abordam esse mesmo tema, ressalta-se que a média de idade dos estudantes universitários 
parece ter sido próxima à encontrada por outros autores (entre 20 e 25 anos)(BUTLER, 2007; CHAMBEL; CURRAL, 2005; COT'TON et al., 2002; MILLER et al., 2008; ROCHFORD et al., 2009; SALAMONSON et al., 2012).

As associações negativas de jornada de trabalho com desempenho acadêmico (HAWKINS et al., 2005; MCKECHNIE et al., 2005; MILLER et al., 2008; ROCHFORD et al., 2009; SALAMONSON et al., 2012; TROCKEL et al., 2000), atitude de estudo (DEROUS; RYAN, 2008), tempo gasto em sala de aula (NAGAI-MANELLI et al., 2012), envolvimento em experiências de aprendizagem extraclasse (FURR; ELLING, 2000), bem-estar (DEROUS; RYAN, 2008) e duração do sono (MILLER et al., 2008) já foram observadas em outros estudos. Também já foram documentadas associações positivas da jornada de trabalho com a percepção, pelos estudantes, de interferência do trabalho no estudo (DEROUS; RYAN, 2008), com a existência de conflito entre o trabalho e o estudo (BUTLER, 2007) e com a percepção de que o trabalho frequentemente afeta, de forma negativa, o progresso acadêmico (FURR; ELLING, 2000). Segundo Trockel et al. (2000), a associação negativa entre a jornada de trabalho e o desempenho acadêmico parece intuitiva: o tempo gasto no trabalho compete com o disponível para o estudo.

A interação da variável jornada de trabalho com outras características do trabalho, entretanto, tem sido menos documentada. A interação entre jornada e demanda no trabalho, que neste estudo não foi significativa no nível de $20 \%$, já foi documentada entre alunos de graduação em Psicologia (DEROUS; RYAN, 2008), quando o desfecho de interesse avaliado foi a atitude de estudo. Tal pesquisa verificou que, entre os alunos com altas demandas no trabalho, os com menor jornada de trabalho apresentaram melhores atitudes de estudo.

No presente estudo, além de ser observada a associação positiva de maior jornada de trabalho com baixo desempenho acadêmico entre alunos com alto controle no trabalho, verificou-se ainda que, entre os alunos com menos de oito horas diárias de trabalho, aqueles com maior controle apresentaram menos chance de baixo desempenho acadêmico. Apesar de essa interação significativa não ter sido evidenciada por outros autores, a associação positiva do controle no trabalho com o desempenho acadêmico (CHAMBEL; CURRAL, 2005), a satisfação com a vida acadêmica (CHAMBEL; CURRAL, 2005) e a facilitação trabalho-estudo (BUTLER, 2007) e a negativa com o conflito entre trabalho e estudo (BUTLER, 2007) e a ansiedade/depressão 
(CHAMBEL; CURRAL, 2005), bem como as associações positivas da autonomia no trabalho com a atitude de estudo e o bem-estar (DEROUS; RYAN, 2008), já foram verificadas em estudos realizados com universitários nos Estados Unidos e em Portugal.

Quanto à demanda no trabalho, corroborando sua associação positiva com o baixo desempenho acadêmico observada neste estudo, outros autores documentaram sua associação negativa com a satisfação com a vida acadêmica (CHAMBEL; CURRAL, 2005) e positiva com o conflito trabalho-estudo (BUTLER, 2007) e com a ansiedade/depressão (CHAMBEL; CURRAL, 2005), também em pesquisas realizadas nos Estados Unidos e em Portugal. Ressaltase que, em um estudo realizado com estudantes universitários australianos, foi observada associação significativa positiva da baixa demanda e do alto controle no trabalho com a satisfação no trabalho, a qual se associou positivamente com o desempenho acadêmico (COTTON et al., 2002).

Assim como neste estudo, que encontrou associação negativa do apoio social no trabalho com o baixo desempenho acadêmico, CHAMBEL e CURRAL (2005) observaram associação positiva do apoio com a satisfação com a vida acadêmica e negativa com a ansiedade/depressão. Esses resultados corroboram a hipótese de que estudantes universitários que percebem seu trabalho como tendo alta demanda, baixo controle e baixo apoio apresentam maior chance de ter um pior desempenho acadêmico.

É fato que pessoas que trabalham 40 horas semanais têm o turno da noite, basicamente, como única opção para cursarem o ensino superior. Dado que o horário comercial é o que disponibiliza mais vagas de trabalho, os que trabalham entre 20 e 40 horas semanais também tendem a optar pelo turno da noite para cursarem a graduação. Estudo (VARGAS; DE PAULA, 2013) revela ainda que, enquanto estudantes das classes econômicas A e B são majoritários nos cursos de graduação em turnos matutinos e integrais, mais de $50 \%$ dos alunos que estudam à noite são das classes econômicas $\mathrm{C}$, $\mathrm{D}$ e E, os quais tendem a precisar contribuir, efetivamente, para a renda familiar total. Vargas e de Paula (2013) destacam ainda que pesquisas realizadas nos cursos superiores nacionais apontam que os estudantes de menor renda familiar são, geralmente, trabalhadores, enquanto os de maior renda familiar não trabalham ou trabalham em tempo parcial, priorizando estágios profissionalizantes, que, por vezes, são não remunerados ou têm baixa remuneração. 
Destaca-se que a remuneração habitual dos estudantes, que na análise de regressão logística simples associou-se positivamente ao baixo desempenho acadêmico, perdeu significância ao ser inserida no modelo múltiplo. Tal perda de significância pode ser explicada pela sua correlação significativa positiva com a jornada diária de trabalho $(r h o=0,574 ; \mathrm{p}<0,01)$ e com a demanda no trabalho $($ rho $=0,276 ; \mathrm{p}<0,01)$, ambas, positivamente associadas ao baixo desempenho acadêmico no modelo múltiplo.

Da mesma forma, o trabalho em instituições de ensino e pesquisa, que na análise de regressão logística simples associouse negativamente com o baixo desempenho acadêmico, perdeu a significância ao ser inserido no modelo múltiplo. As variáveis intermediárias na associação entre trabalho em instituições de ensino e pesquisa e desempenho acadêmico identificadas neste estudo foram jornada, demanda, controle e apoio social no trabalho. Assim, podese justificar tal resultado com base no achado de que, segundo o teste não paramétrico de Mann-Whitney, a jornada e a demanda média são significativamente menores, enquanto o controle e o apoio social médio são significativamente maiores entre os alunos que trabalham em instituições de ensino e pesquisa, quando comparados aos estudantes que trabalham em outras instituições.

Ao analisarem a estrutura da representação social de estudantes brasileiros sobre temas relacionados ao trabalho e ao estudo, pesquisas apontaram que o termo "trabalho" traz, predominantemente, significados positivos, relacionados a ganhos econômicos, psicossociais, morais, à construção de um futuro/experiência profissional, a uma melhor inserção no mercado de trabalho; enquanto, para "trabalhar e estudar", predominam significados negativos (cansaço, falta de tempo, prejuízo ao estudo, sono), como expressão da sobrecarga derivada da junção de duas atividades que demandam esforço e que são desenvolvidas competitivamente. A forma contraditória como os estudantes estruturam a relação trabalho-estudo fica evidente nessas pesquisas (FISCHER et al., 2003; OLIVEIRA et al., 2001; OLIVEIRA et al., 2005). Logo, a influência do trabalho no estudo e no desempenho acadêmico deve ser alvo de análise/ações por profissionais comprometidos com a educação, desde o ciclo básico até o Ensino Superior, na busca por melhores condições de aprendizado para os estudantes trabalhadores.

De posse dessas evidências científicas sobre as características do trabalho/estágio que prejudicam ou beneficiam o desempenho acadêmico, os docentes do Ensino Superior podem fomentar 
discussões em sala de aula, ampliar o acesso a essas evidências e motivar uma escolha consciente pelos alunos, sujeitos também ativos nesse processo (TROCKEL et al., 2000). Podem tanto buscar uma mudança na política de estágio da universidade (SALAMONSON et al., 2012) quanto desenvolver abordagens/estratégias em suas disciplinas para identificar os alunos em condições de trabalho/ estágio que os deixem mais vulneráveis a um pior desempenho acadêmico (como maiores jornadas e demandas e menores controle e apoio social no trabalho), dando a eles maiores estímulo, incentivo e apoio, para explorar suas potencialidades, fomentar seu aprendizado/desempenho acadêmico e reduzir o abandono do curso (TROCKEL et al., 2000). Além disso, não pode ser ignorada a necessidade de criação de uma legislação específica para a situação desses estudantes trabalhadores, a qual deve ser discutida à luz de exemplos concretos adotados em outros países, como Portugal e Cuba (VARGAS; DE PAULA, 2013).

Por fim, cabe ressaltar que o fato de ter sido utilizada uma amostra de conveniência pode ter introduzido um viés de seleção neste estudo. Tal limitação também foi enfrentada por outros autores que utilizaram amostras de conveniência (BUTLER, 2007; DEROUS; RYAN, 2008; HAWKINS et al., 2005; NAGAI-MANELLI et al., 2012; ROCHFORD et al., 2009) ou tiveram taxas de resposta inferiores a 50\% (COTTON et al., 2002; ROCHFORD et al., 2009).

Não obstante, os resultados sugerem que características do trabalho/estágio podem prejudicar ou beneficiar o desempenho acadêmico. Logo, destaca-se a necessidade de atenção dos alunos e dos docentes do Ensino Superior na seleção de ambientes de trabalho/ estágio favoráveis (com menores jornada e demanda e maiores controle e apoio social), pela potencialidade deles em promover um melhor desempenho acadêmico.

\section{AGRADECIMENTOS}

Ao CNPq (501766/2007-3; 500782/2008-3; 472153/2006-4; 307919/2006-4), à CAPES, à FAPESP (07/04648-4; 06/59053-2) e ao PIBIC-CNPq, pelo apoio financeiro recebido.

\section{REFERÊNCIAS}

ALVES, M. G. de M. et al. Short version of the "job stress scale": a Portuguese-language adaptation. Revista de Saúde Pública, São Paulo, v. 38, n. 2, p. 164-171, abr. 2004. 
BUTLER, A. B. Job characteristics and college performance and attitudes: A model of workschool conflict and facilitation. Journal of Applied Psychology, v. 92, n. 2, p. 500-510, mar. 2007. CHAMBEL, M. J.; CURRAL, L. Stress in academic life: Work characteristics as predictors of student well-being and performance. Applied Psychology, v. 54, n. 1, p. 135-147, 2005.

COTTON, S. J.; DOLLARD, M. F.; DE JONGE, J. Stress and student job design: Satisfaction, well-being, and performance in university students. International Journal of Stress Management, v. 9, n. 3, p. 147-162, 2002.

DEROUS, E.; RYAN, A. M. When earning is beneficial for learning: The relation of employment and leisure activities to academic outcomes. Journal of Vocational Behavior, v. 73, n. 1, p. 118-131, 2008.

DOCHY, F. et al. The Use of Self-, Peer and Co-assessment in Higher Education: A review. Studies in Higher Education, v. 24, n. 3, p. 331-350, 1999.

FISCHER, F. M. et al. Efeitos do trabalho sobre a saúde de adolescentes. Ciência e Saúde Coletiva, v. 8, n. 4, p. 973-984, jan. 2003.

FURR, S. R.; ELLING, T. W. The Influence of Work on College Student Development. NASPA Journal, v. 37, n. 2, p. 454-70, 2000.

HAWKINS, C. A. et al. The relationships among hours employed, perceived work interference, and grades as reported by undergraduate social work students. Journal of Social Work Education, v. 41, n. 1, p. 13-27, Winter. 2005.

KARNILOWICZ, W. A comparison of self-assessment and tutor assessment of undergraduate psychology students. Social Behavior and Personality, v. 40, n. 4, p. 591-604, 2012. LÓPEZ-PASTOR, V. M. et al. Students' self-grading, professor's grading and negotiated final grading at three university programmes: analysis of reliability and grade difference ranges and tendencies. Assessment \& Evaluation in Higher Education, v. 37, n. 4, p. 453-464, jun. 2012.

MCKECHNIE, J.; DUNLEAVY, K.; HOBBS, S. Student employment and its educational impact: a scottish study. Scottish Educational Review, v. 37, n. 1, p. 58-67, 2005.

MILLER, K.; et al. Relationship of work hours with selected health behaviors and academic progress among a college student cohort. Journal of American College Health, v. 56, n. 6, p. 675-679, 2008.

NAGAI-MANELLI, R. et al. Sleep length, working hours and socio-demographic variables are associated with time attending evening classes among working college students. Sleep and Biological Rhythms, v. 10, n. 1, p. 53-60, 2012.

NULTY, D. D. Peer and self-assessment in the first year of university. Assessment $\mho$ Evaluation in Higher Education, v. 36, n. 5, p. 493-507, 2011.

OLIVEIRA, D. C. de. et al. Futuro e liberdade: o trabalho e a instituição escolar nas representações sociais de adolescentes. Estud. psicol., Natal-RN, v. 6, n. 2, p. 245-258, dez. 2001.

Adolescência, trabalho e estudo: análise comparativa das representações sociais de adolescentes trabalhadores e não trabalhadores. Rev. Enferm, Rio de Janeiro, UERJ, v. 13, n. 2, p. 229-237, ago. 2005.

ROCHFORD, C.; et al. Paid part-time employment and academic performance of undergraduate nursing students. Nurse education today, v. 29, n. 6, p. 601-606, ago. 2009.

SALAMONSON, Y. et al. The impact of term-time paid work on academic performance in nursing students: a longitudinal study. International journal of nursing studies, v. 49, n. 5, p. 579-585, mai. 2012. 
TROCKEL, M. T.; et al. Health-related variables and academic performance among firstyear college students: implications for sleep and other behaviors. Journal of American college health, v. 49, n. 3, p. 125-131, nov. 2000.

VARGAS, H. M.; DE PAULA, M. F. C. A inclusão do estudante-trabalhador e do trabalhadorestudante na educação superior: desafio público a ser enfrentado. Avaliação, Campinas-SP, v.18, n. 2, p.459-485, jul. 2013.

Recebido: $19 / 08 / 2013$

Aprovado: 09/10/2014

Contato:

Rua Leopoldo Bulhões, 1480

Pavilhão Primeiro de Maio - sala 17 Manguinhos

Rio de Janeiro $\mid$ RJ $\mid$ Brasil

CEP 21.041-210. 
